\title{
An Assessment of the Application of Bernoulli's Theorem in the Generation of Lift Force
}

\author{
Garv Shah ${ }^{1}$, Aman Singhal $^{2}$, Raadnya Apte $^{3}$, Rushaad Dupetawalla \\ ${ }^{1}$ The Cathedral and John Connon School, Mumbai, India \\ ${ }^{2} \mathrm{KC}$ College, Mumbai, India \\ ${ }^{3}$ Pace Junior Science College, Mumbai, India \\ \#Advisor
}

\section{ABSTRACT}

In this paper, we will be performing a detailed analysis of the application of Bernoulli's Theorem in aviation and aerodynamics. The aim of our experiment and consequently this paper is to verify the application of Bernoulli's Theorem in the aviation industry. In the field of aerodynamics, Bernoulli's Theorem has been specifically used in shaping the wings of an aircraft. Over the years, however there has been a significant controversy in the aviation industry regarding the generation of lift force, especially the applicability of Newton's Third Law of Motion along with Bernoulli's Theorem [1]. The controversy seems to be due to a combined effect of Newton's and Bernoulli's theorems' (e.g., 'Equal Transit Time Theory' [2]), which may be incorrectly applied in the real world. Further, it seems that people are over-simplifying the problem of aerodynamic lift leading to the dismissal of either one of the theorems, when in reality both the theorems seem to be at play, as explained in this paper. For the generation of lift in air, momentum, mass and energy need to be conserved. Newton's laws take into account the conservation of momentum, whereas Bernoulli's Theorem considers the conservation of energy. Hence, they are both relevant for the generation of lift in air [1]. However, no one has been able to determine accurately the working of both these theorems in the process of providing lift to an aircraft [3]. Through this research paper, we have been able to prove the effect of Bernoulli's Theorem in generating lift in air.

\section{Introduction}

Bernoulli's Theorem is a theorem used in fluid dynamics. It was originally given by the famous physicist Daniel Bernoulli in the year 1738 in his book 'Hydrodynamica' which eventually formed the base of fluid mechanics and fluid dynamics in the following years [1]. Bernoulli's principle states that "When an incompressible and nonviscous fluid (for example air) flows in a stream-lined motion from one place to another, then at every point of its path, the sum of the pressure energy, potential energy and kinetic energy (i.e. total energy) per unit volume remains constant." [4]

The four forces which are responsible for flight of an aircraft are lift, weight, thrust and drag. Lift is the force which initially helps the aircraft takeoff by gaining the necessary elevation. After takeoff, lift also helps in keeping the aircraft airborne by balancing its weight. Weight or gravity is the force which constantly tries to pull the aircraft back to the ground. It is unable to do so for most duration of the flight since the lift either exceeds the weight or counteracts it. Thrust is that force which assists in moving the aircraft ahead thereby allowing it to cover the required distance. Drag or air resistance is that force which tries to oppose the tendency of motion of the aircraft by trying to pull it in the opposite direction of the impending motion.

IWhen an aircraft takes off, the two forces which assist it are lift and thrust. The pilot has to increase the lift of the aircraft so much so that it exceeds its weight, thereby helping it to ascend. This is primarily done using three techniques. First, the speed is increased by activating the engines on full throttle. Second, the angle of attack is increased to about $10^{\circ}-15^{\circ}$ [5] (Figure 1). The angle of attack is the angle that the fuselage (aircraft's body) 
forms with the horizontal. The angle of attack directs the air downwards (action) and hence, by Newton's Third Law of Motion ("to every action, there is an equal and opposite reaction"), an upward reaction force is created on the plane, which is the lift force. Third, the flaps and the slats are activated to further force air downwards, thus creating more lift. These three techniques help in getting the aircraft airborne. After the aircraft has reached the desired altitude, the angle of attack becomes approximately $2^{\circ}$, which alone cannot create sufficient lift [6]. But lift is still required for the aircraft to balance its weight otherwise it would plummet to the ground. Our hypothesis is that the lift at a constant altitude is generated in accordance with Bernoulli's Theorem.

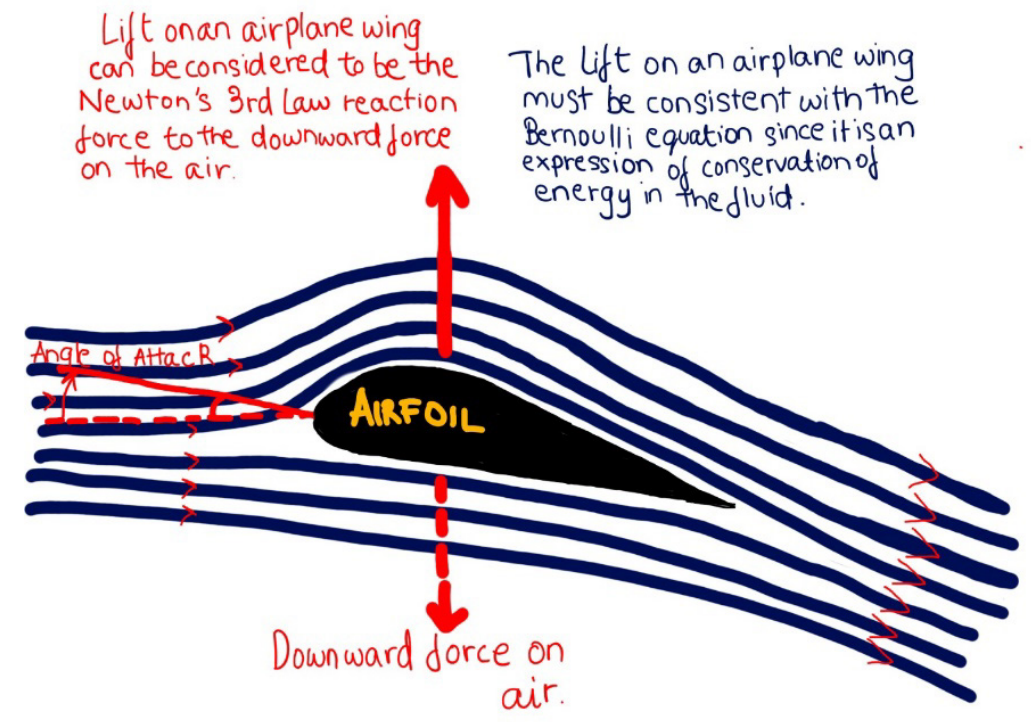

Figure 1 shows how the angle of attack forces air downwards, thus creating an upward reaction force.

Bernoulli's Equation: [4]

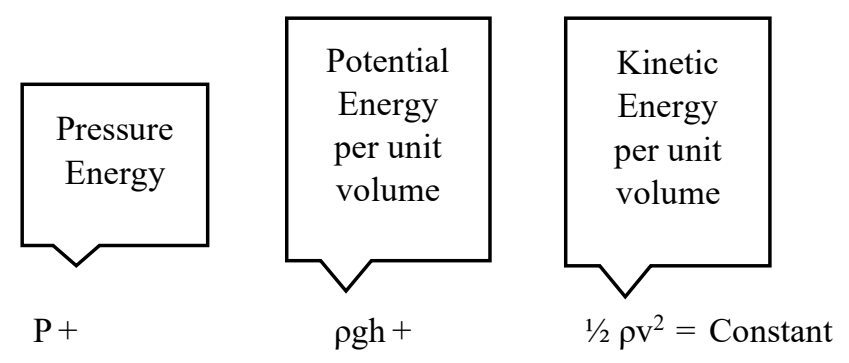

$\rho$ : Density of fluid, g: Acceleration due to gravity, h: Height of fluid, v: Velocity of fluid

Deriving the Lift Formula: [4]

In accordance with Bernoulli's theorem, we can say:

$$
\mathrm{P}_{1}+\rho g \mathrm{gh}_{1}+1 / 2 \rho \mathrm{v}_{1}^{2}=\mathrm{P}_{2}+\rho \mathrm{gh}_{2}+1 / 2 \rho \mathrm{v}_{2}^{2}
$$

However, $\mathrm{h}_{1} \approx \mathrm{h}_{2}$ since both the wing surfaces in an aircraft will be at the same altitude.

$$
\begin{gathered}
\text { Therefore, } P_{1}+\rho g h_{1}+1 / 2 \rho v_{1}^{2}=P_{2}+\rho g h_{z}+1 / 2 \rho v_{2}^{2} \\
P_{2}-P_{1}=1 / 2 \rho\left(v_{1}^{2}-v_{2}^{2}\right)
\end{gathered}
$$




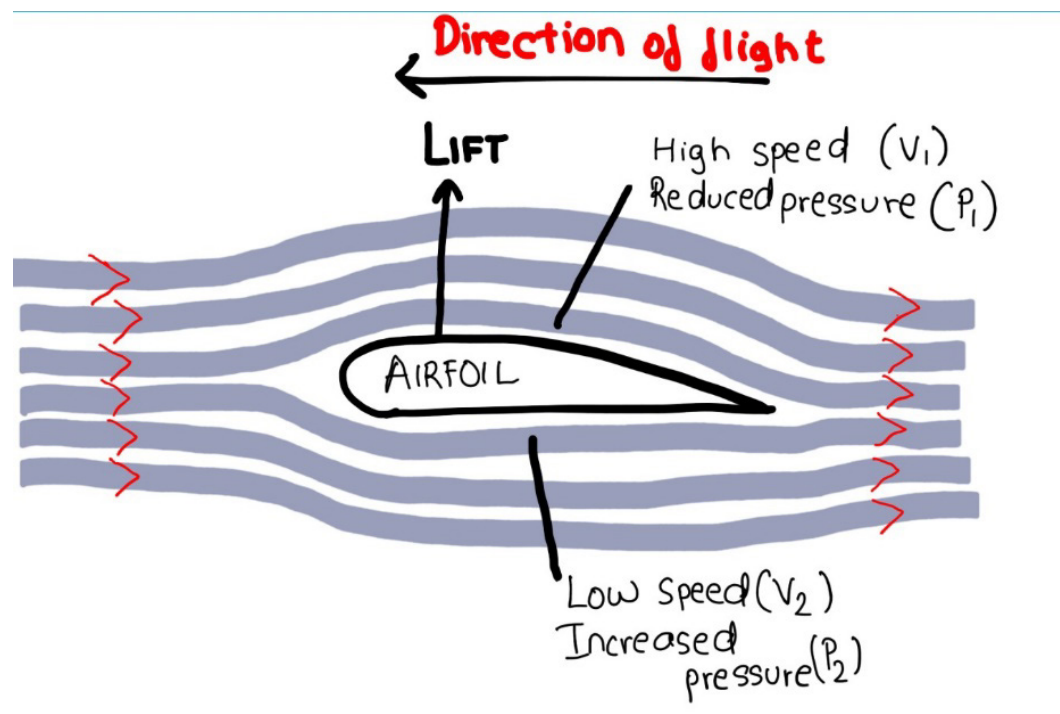

Figure 2 shows the wing at a constant altitude, with the low and high pressure regions created.

$$
\text { Therefore, } \begin{aligned}
F_{\text {net }} & =P_{2} A-P_{1} A, A: \text { Surface area of wing } \\
& =1 / 2 \rho_{\text {air }} A\left(v_{1}^{2}-v_{2}^{2}\right)
\end{aligned}
$$

$\approx$ Lift force generated on the wing (Figure 2)

The wings of most aircrafts are designed so that the curvature of their upper surface is greater than that of their lower surface and the front end is shaped round while the back end is flattened (airfoil). When the air flow hits the front end of the wing, it splits. The air which passes over the top of the wing, sticks to the top of the wing and moves along its curvature. This is known as the 'Coandă Effect' [7]. Now, because of the curvature of the wing, the velocity of air on the upper surface is higher than the velocity of air on the lower surface, due to which a lower pressure region is created above the wing and higher pressure region is created below the wing. This difference in the pressure $\left(\approx 1 / 2 \rho_{\text {air }} \mathrm{A}\left(\mathrm{v}_{1}{ }^{2}-\mathrm{v}_{2}^{2}\right)\right)$ essentially provides the lift force. This lift force produced accounts for the weight of the aircraft thereby allowing it to cruise at a constant altitude. Now if the aircraft needs to increase its altitude further (i.e. after it has already reached cruising altitude) it again has to increase the angle of attack; hence Newton's Third Law again comes into play and Bernoulli's Theorem also has some effect during this stage.

During landing, the aircraft needs a high drag, low thrust and most importantly, it needs to pitch its nose upwards. This is required to greatly reduce the aircraft's cruising velocity, to slightly above the stall speed and to make a gentle landing on the runway. This is known as the 'Flare Maneuver' [8]. The high drag is achieved by activating the flaps and slats. The flaps and slats now play an opposite role than the one during takeoff. They now force the air upwards thereby increasing drag and subsequently reducing the aircraft's altitude and allowing it to descend comfortably. The pitch is achieved by increasing the angle of attack slightly while descending, to the extent that only the nose of the plane tilts upwards, while the altitude goes on decreasing. Hence, we always see the rear wheels of the aircraft touchdown before the front wheels. 


\section{Experiment}

In order to test the hypothesis, we made the fuselage of a glider using depron, and two wings - a flat, thin,

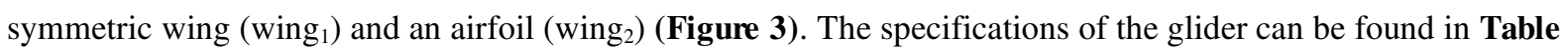

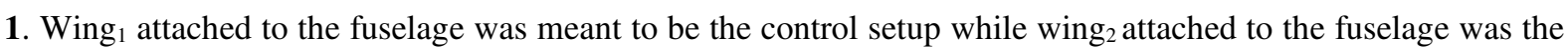
experimental setup. We chose an open field as the site of the experiment and a windless day in order to ensure the

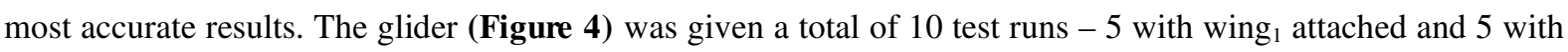
wing $_{2}$, always from the same starting point, by the same person and at the same initial angle of attack.

\begin{tabular}{|c|c|c|}
\hline Table 1 & Glider with wing & Glider with wing $_{2}$ \\
\hline Wingspan & $940 \mathrm{~mm}$ & $\approx 193 \mathrm{~g}$ \\
\hline Weight & $\approx 182 \mathrm{~g}$ & $=$ \\
\hline Centre of Gravity & $\begin{array}{c}38-44 \mathrm{~mm} \text { from leading edge of the } \\
\text { wing }\end{array}$ & \\
\hline
\end{tabular}

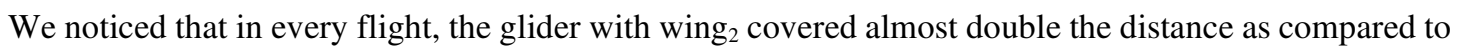

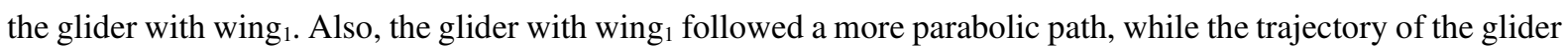
with wing $_{2}$ was flatter. The mean distance travelled by the glider with wing ${ }_{1}$ was approximately 11.0 meters (error margin $- \pm 0.26 \mathrm{~m}$ ) while the glider with wing ${ }_{2}$ covered a mean distance of approximately 19.8 meters (error margin $- \pm 0.56 \mathrm{~m})$. 


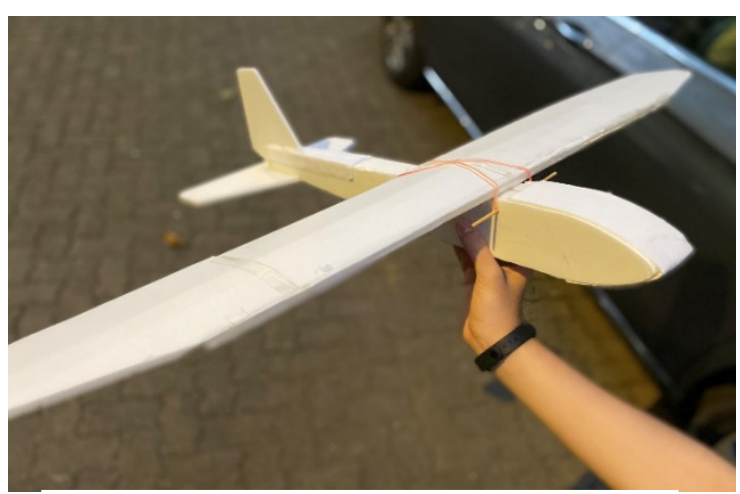

Figure $\mathbf{4}$ is the glider used in the experiment with the airfoil attached. The curvature is clearly visible.

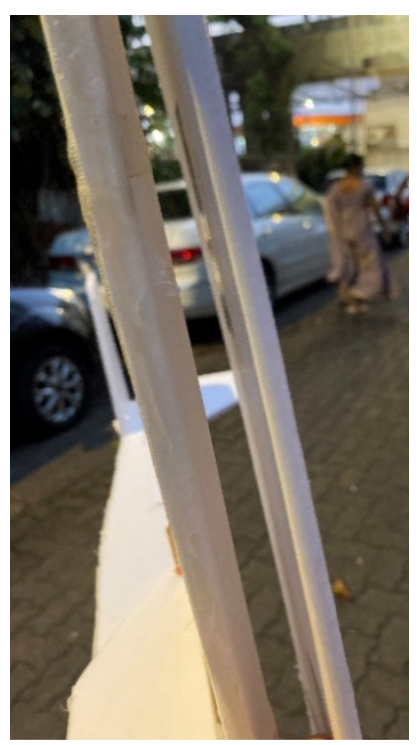

Figure 3 is a comparison of the cross section of the two wings. The one on the right is wing wnd on the left is wing $_{2}$.

\section{Discussion}

We hypothesized that the glider with an airfoil wing, in accordance with Bernoulli's Theorem, would cover a larger distance as compared to the glider with an exactly symmetric wing. The data collected from the above experiment (Figure 5) is in accordance with our hypothesis. However, we did not expect the difference in distance flown to be that great. It just goes to show the significance of Bernoulli's effect in providing lift to the glider. 


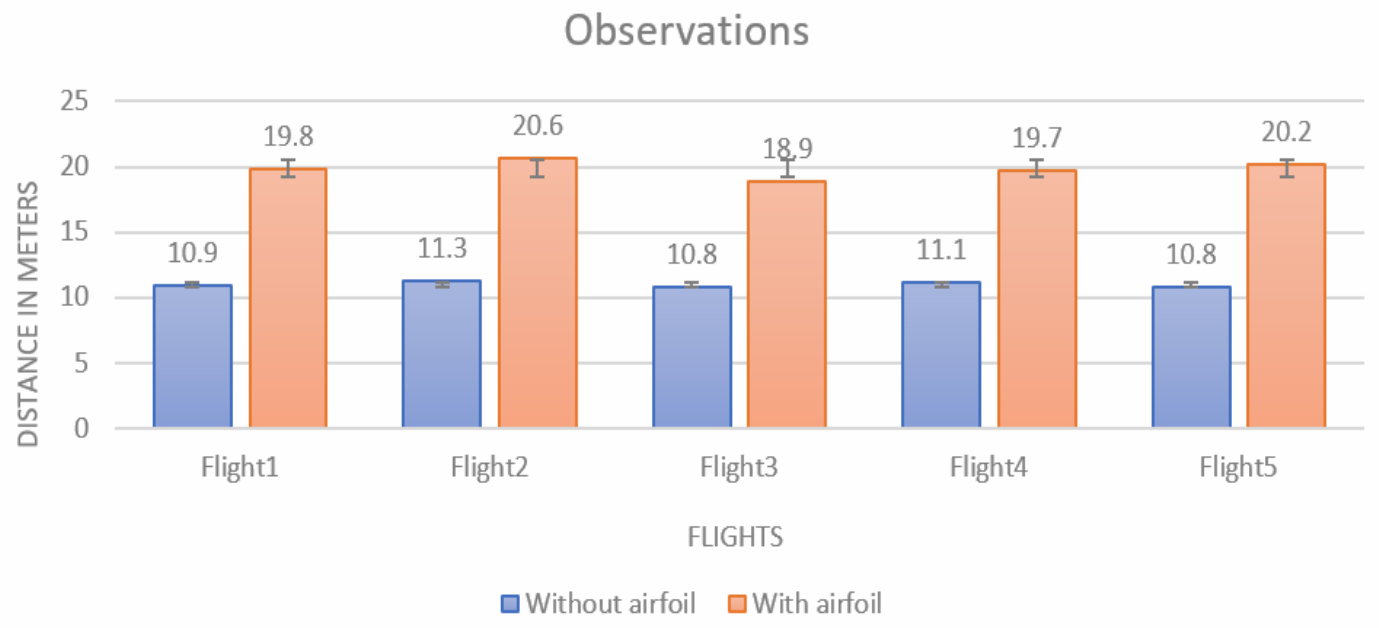

Figure 5 represents the data collected from the experiment and clearly shows the difference in distance flown.

The trajectory of the glider can be explained as follows: the somewhat parabolic trajectory of the glider

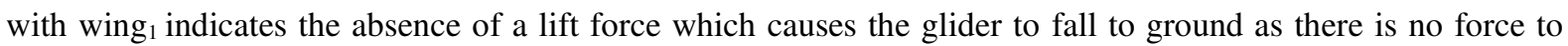
counteract its weight. Whereas, in case of the glider with wing $_{2}$, the curvature in the upper surface of the wing would create a lower pressure area above the wing and a higher pressure area under the wing. This difference in pressure provides some lift to the wing and hence the trajectory is flatter and the glider remains airborne for a longer period of time, after which it falls to the ground because of its weight and absence of thrust.

Bernoulli's theorem is largely correct except for one thing: it has been experimentally observed that air flows faster above a curved surface, however, Bernoulli's theorem is unable to explain why [3], and no one has been able to provide an explanation with absolute surety; but we cannot disregard Bernoulli's theorem for this

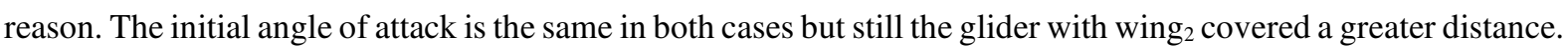
The only difference in the wings is their curvature. Hence, we can conclusively say that there has to be some effect of Bernoulli's theorem mid-flight, in the glider with wing 2.

\section{Limitations}

Our experiment is unable to prove the effect of Newton's Third Law as the angle of attack is same in the control and experimental setup, but does indicate the effect of Bernoulli's theorem. We tried to minimize natural factors and human error as much as possible, but they might have had a miniscule effect on the observations. We were able to prove that Bernoulli's Theorem had some effect, but with more resources, we could have collected more data including the instantaneous velocity, wind speed, etc.

\section{Conclusion}

Through this paper, we have tried to give some proof regarding the application of Bernoulli's Theorem in the generation of lift force for an aircraft. However, our intent is not to be dismissive of the role of Newton's Third Law of Motion at any stage of the process. Instead, we believe that the whole science behind the flight of an aircraft lies behind the cohesive working of these two principles in their respective stages. The magnitude of the effect of these two principles differs in different stages of flight, for example, we believe that Newton's Third Law of Motion superimposes Bernoulli's Theorem during the takeoff and landing procedures while Bernoulli's 
Theorem largely comes into play during the cruising period of the flight. We are also of the opinion that the science of air travel is fairly subjective and enigmatic.

\section{Acknowledgments}

We would like to take this opportunity to express our heartfelt gratitude and sincere thanks to our parents. They have supported us through the entire course of this research paper; right from organizing the necessary materials to giving us honest and critical views about our work. Without their unwavering support, we wouldn't have been able to complete this paper in the stipulated period of time.

\section{References}

1. Nancy Hall, "Bernoulli and Newton”, National Aeronautics And Space Administration Glenn Research Centre (NASA-GRC), May 13, 2021. Retrieved from: https://www.grc.nasa.gov/www/k12/airplane/bernnew.html\#: :text=There $\% 20 \mathrm{is} \% 20$ also $\% 20 \mathrm{an} \% 20$ incorrect,of $\% 20 \mathrm{a} \% 20 \mathrm{flow} \% 20$ of $\% 20$ gas

2. Professor Holger Babinsky, "How wings really work", Department of Engineering, University of Cambridge, May 29, 2012. Retrieved from: http://www.eng.cam.ac.uk/news/how-wings-really-work

3. Nick Higgins, "No One Can Explain Why Planes Stay in the Air", Scientific American, February 1, 2020. Retrieved from: https:/www.scientificamerican.com/article/no-one-can-explain-why-planes-stay-in-the-air/

4. "Nootan Class 11 ISC Physics Part 2” by Raj Kumar, G. L. Mittal and Tarun Mittal. Published by: "Nageen Prakashan Pvt. Ltd."

5. "How do Airplanes fly?", Lesics, October 31, 2018. Retrieved from: https://www.youtube.com/watch?v=F077WDnB8P8

6. Perspective - Barb Claitman, “AOA MEASUREMENT”, Boeing. Retrieved from: https://www.boeing.com/commercial/aeromagazine/aero 12/attack story.html

7. Engr Fahad, "How aeroplanes fly? Aeroplane Design and Different Parameters Explained", Electronic Clinic, January 31, 2021. Retrieved from: https://www.electroniclinic.com/tag/how-aeroplanes-fly/

8. Matt Claiborne, “The Landing Flare: How Pilots Can Improve Their Landing”, Aero Corner. Retrieved from: https://aerocorner.com/blog/the-landing-flare-or-roundout/ 